Mots. Les langages du politique

$70 \mid 2002$

La politique en chansons

\title{
Deux grands hymnes idéologiques : le Te Deum, l'Internationale
}

Two ideological anthems: Te deum and the Internationale

Dos grandes himnos ideológicos : el Te deum, la Internacional

Juana Ugarte

\section{CpenEdition}

Journals

Édition électronique

URL : https://journals.openedition.org/mots/8923

DOI : $10.4000 /$ mots.8923

ISSN : 1960-6001

Éditeur

ENS Éditions

Édition imprimée

Date de publication : 1 novembre 2002

Pagination : $9-27$

ISBN : 2-84788-016-X

ISSN : 0243-6450

\section{Référence électronique}

Juana Ugarte, « Deux grands hymnes idéologiques : le Te Deum, l'Internationale », Mots. Les langages du politique [En ligne], 70 | 2002, mis en ligne le 07 mai 2008, consulté le 23 avril 2022. URL : http:// journals.openedition.org/mots/8923; DOI : https://doi.org/10.4000/mots.8923

(c) ENS Éditions 
Juana $\mathrm{UGARTE}^{\circ}$

\section{Deux grands hymnes idéologiques : le Te Deum, l'Internationale}

Chants révolutionnaires, hymnes patriotiques et marches militaires sont au cœur du dispositif scénographique du pouvoir et des luttes pour sa conquête.

Alain Darré ${ }^{1}$

Le Te Deum et 1'Internationale, très séparés par leur époque de création, puisque l'un date du $4^{\mathrm{e}}$ siècle et l'autre du $19^{\mathrm{e}}$, et par la langue utilisée (le latin et le français au départ), sont devenus par des voies ellesmêmes différentes deux hymnes historiques institutionnalisés, le premier dans le livre du Pontifical Romain (le Liber Pontificalis du $15^{\mathrm{e}}$ siècle) et le second dans les usages de la $2^{\mathrm{e}}$ et de la $3^{\mathrm{e}}$ Internationales. Ces chants majeurs ont traversé le temps, liés de plus en plus fortement à la parole de pouvoir, qu'il s'agisse du pouvoir en place ou de sa contestation, autre pouvoir dans une autre place. Et cela n'est pas révolu. Les voilà contemporains : aux deux bouts de l'ancien empire de Charles Quint, en l'an 2000, le roi de Belgique assiste à un Te Deum dans la cathédrale de Bruxelles le jour de la Fête nationale et, la même année, le Parti Socialiste Ouvrier Espagnol clôt son congrès en chantant l'Internationale.

Certes les conditions d'élaboration et de diffusion de ces deux hymnes sont très différentes, spécifiques aux époques et aux milieux de création, mais, dans la reprise politique de ces chants séculaires - musique et paroles -, plusieurs traits les caractérisent ensemble comme hymnes idéologiques. N'ont-ils pas sauté les frontières pour servir à de multiples actes politiques? N'obéiraient-ils pas à une même loi de structure, naissant et se

- Faculté d'Histoire, Campus des Humanités El Milan, Université d'Oviedo, Espagne.

1. "Prélude. Pratiques musicales et enjeux de pouvoir», dans Musique et Politique. Les répertoires de l'identité, Rennes, Presses Universitaires de Rennes, 1996, p. 15. 
propageant au sein d'une communauté réunie autour d'un fort nous identitaire?

Pour illustrer cette double problématique, nous évoquerons trois directions de recherche : l'ancrage énonciatif de la parole de pouvoir dans l'expression du nous pluriel; la représentation discursive qui émerge de cet acte énonciatif; enfin les référents repris, remotivés et rattachés historiquement par l'usage politique au nous communautaire.

\section{Quand la parole de pouvoir prend le nous de la communauté}

Qui parle dans ces hymnes, et pourquoi? Dans quelles conditions se sont effectuées leur naissance (auteurs, texte, musique, chanteurs et récepteurs initiaux) et leur transmission : comment s'est réalisée l'extension du nous avec l'expansion du chant?

L'analyse du Te Deum achoppe, vu l'éloignement des sources, sur la difficulté d'identification de l'auteur. On l'a successivement attribué à saint Ambroise, à saint Augustin, à saint Hilaire, puis à Nicetes de Remessiane : ses origines sont donc controversées ${ }^{2}$. Mais cette analyse met en œuvre une logique qui opère à l'intérieur du texte. On pense aujourd'hui qu'il fusionnerait plusieurs apports : des versets primitifs ou psaumes anciens d'une part et, d'autre part, des versets qui leur ont été associés après le concile de Nicée de l'année 325 et qui consacrent la reconnaissance du mystère trinitaire (ajout du filioque au Père et à l'Esprit). C'est pourquoi, dans la liturgie catholique, le Te Deum est considéré comme un message doxologique, l'Hymnus trinitatis. À son origine donc, il s'agit d'un récitatif (avec peut-être un refrain qui a disparu) visant au rassemblement d'une communauté spirituelle et sociale (les chrétiens de l'époque, séparés des ariens partisans de la seule humanité du Christ). C'est le crédo identitaire d'une Ecclesia ( Sancta confitetur Ecclesia »).

L'analyse de la transmission met en relief l'usage du Te Deum, au $4^{\mathrm{e}}$ siècle, parmi les chants d'Office de la liturgie des monastères, où il est consacré dans les Règles monastiques. Ainsi la liturgie monacale du Livre d'Heures l'inclut dans les Matines ou les Vêpres tout au long du MoyenÂge. La liturgie celtique l'utilise comme chant eucharistique pendant la messe («Hymnus quando communicant sacerdotes »), cependant que,

2. M. Kunzler, 1997, La liturgie de l'Église. Manuel de théologie catholique, Luxembourg, Éditions Saint Paul, vol. 10; 1997, La liturgie. Encyclopédie de la Foi, Clovis; M. Righetti, 1955, Historîa de la liturgia, Madrid, BAC. 
dans l'Eglise de Rome même, à Saint-Pierre, apparait un usage ancien et particulier qui se rapporte aux anniversaires des Papes.

On croit savoir que sa transmission fut véhiculée à l'origine par ce qu'on appelle le « vieux chant romain » qu'a suivi le « chant grégorien » dont la fonction était de mettre en valeur le pouvoir de la parole, reconnue parole d'Église ${ }^{3}$. Ce primat de la parole explique l'air monocorde sur lequel elle s'appuie, plain-chant, sans guère de distance entre des notes elles-mêmes sans mesures, sur un rythme très lent, composé de nombreuses liaisons d'expression (au nombre de 129) entre des figures musicales faites pour conférer continuité et force à l'aspect récitatif qui l'apparente à une litanie. La discrétion du musical le soumet à la parole, à son action d'endoctrinement et de communion, à sa fonction mnémonique aussi, puisque sa prose rimée convenait à l'origine à une transmission orale. Scénographie de solennité au service d'une Parole sacrée. À partir de partitions modernes, nous faisons figurer dans le tableau suivant une comparaison des mots, des notes et figures musicales du Te Deum (en exceptant, bien entendu, le Miserere final ajouté postérieurement) et de l'Internationale :

\begin{tabular}{|c|c|c|}
\hline & Te Deum & Internationale \\
\cline { 2 - 3 } - nombre de formes linguistiques & 130 & 300 \\
- nombre de notes de musique & 487 & 121 \\
dont : blanches & 0 & 17 \\
blanche pointée & 0 & 1 \\
noires & 260 & 38 \\
croches & 227 & 61 \\
doubles croches & 0 & 4 \\
- nombre de liaisons d'expression & 129 & 0 \\
- nombre de versets/vers & 31 & 52 \\
\hline
\end{tabular}

Tableau : Signes linguistiques et musicaux des deux hymnes

La valeur d'une telle comparaison est évidemment toute relative, ne serait-ce qu'à cause de la notation (même les neumes n'avaient pas la précision de la gamme dite d'Arezzo et ce n'est qu'au $12^{\mathrm{e}}$ siècle qu'est notée

3. A. Coueur Clevey, 1998, Histoire du langage musical occidental, Paris, PUF; F. Sapey, 1999, Histoire de la musique en Europe, Paris, PUF. 
la durée des sons) et du type de langue. Mais, outre l'écart entre une absence de mesure et un rythme très scandé, outre aussi le fait que les notes du Te Deum jouent à l'intérieur d'une seule octave alors que celles de l'Internationale dépassent largement l'octave, ce tableau comparatif a pour intérêt de montrer l'opposition entre le style «coulé » et monotone (tout se joue entre noires et croches) du chant religieux et la plus grande variété des différences de longueur non tempérées par des liaisons d'expression qui caractérise l'hymne révolutionnaire.

L'analyse de l'Internationale dans son contexte social concerne d'abord la personnalité de l'auteur des paroles, le socialiste Eugène Pottier, fils d'un artisan parisien, emballeur, pion, commis-papetier, dessinateur sur indienne puis patron d'un atelier de dessin industriel, goguettier et poète ouvrier quarante-huitard puis communard. Les historiens ${ }^{4}$ l'ont décrit comme intimement associé aux luttes révolutionnaires du $19^{\text {e }}$ siècle, sensible aux révoltes des canuts, présent sur les barricades en février et juin 1848 puis sur celles de la Commune de Paris, dont il a été membre du Comité central au titre du $2^{\mathrm{e}}$ arrondissement. Du fouriérisme et du babouvisme de sa jeunesse, il vire à l'anarchisme, signe le Manifeste de la Commune, compose des appels aux armes contre les Versaillais et participe au combat. Condamné à mort, il échappe à la répression. C'est pendant cette traque, avant sa fuite vers l'exil anglais puis américain, qu'il a, dit-on, composé dans une mansarde parisienne l'Internationale (sur l'air de la Marseillaise, comme bien d'autres chants politiques du temps). «L'Internationale est l'œuvre d'un proudhonien doublé d'un vaincu » ${ }^{5}$. Le poème, daté de juin 1871 et dédié à Gustave Lefrançais, instituteur anarchiste, concentre tout le désespoir révolté de cette époque; il est surtout l'expression d'une volonté permanente chez Pottier de communier avec militants et masses ouvrières (n'a-t-il pas créé avec Courbet une Fédération des Artistes communards, adhéré à l'Internationale, au Socialist Labor Party, à la Loge des Égalitaires, à des mouvements mutuel-

4. M. Ferro, 1996, L'Internationale. Histoire d'un chant de Pottier et Degeyter, Paris; M. Dommanget, 1971, Eugène Pottier, membre de la Commune et chantre de l'Internationale, Paris, Édi; E. Tersen, 1962, L'Internationale, Paris, Éditions sociales; M. Vovelle, « La Marseillaise », dans P. Nora (dir.), 1992, Les lieux de mémoire, Paris, Gallimard; J. Estager, G. Bossi, 1988, L'Internationale, 1888-1988, Paris, Messidor; P. Brochon, 1966, Eugène Pottier, ouvrier, poète, communard, auteur de l'Internationale. Euvres complètes, Paris, F. Maspero; M. Choury, 1970, Les poètes de la Commune, Paris, Seghers; E. Museux, Eugène Pottier, dans Portraits d'hier, 32, 1 ${ }^{\mathrm{er}}$ juillet 1910.

5. P. Brochon, 1966, p. 17. 
listes?). Conçu comme hymne d'unité ouvrière, l'Internationale s'inscrit dans la dynamique antisectaire de rassemblement qui fait que, à la veille de mourir, Pottier dédie encore son En avant la classe ouvrière! au " parti révolutionnaire cosmopolite de toutes les écoles ${ }^{6}$.

Pourtant, la transmission du chant n'a pas d'abord passé par les organisations de lutte mais par une goguette politique, la Lice chansonnière et surtout par la publication, grâce à Rochefort, de ses Chants révolutionnaires, qui, quelques mois avant sa mort (1887), relance l'œuvre de Pottier. Dix-mille personnes ont beau, malgré la police, se rassembler autour de la bière aux cris de «Vive Pottier, Vive la Commune!», la gloire lui a pourtant échappé. Elle s'est reportée sur son hymne. Car c'est l'année suivante que la Lyre des travailleurs, chorale lilloise du Parti Ouvrier Français (Pottier avait poussé Guesde et Lafargue à la création du POF) demande à l'un de ses membres, Pierre Degeyter, un tourneur sur bois, de mettre en musique certains des Chants révolutionnaires. Seconde naissance : l'air de l'Internationale est composé, le soir même, sur un harmonium, chanté par son frère Adolphe, baryton, aussitôt repris dans une fête syndicale, publié à Lille dès 1889 , paroles et musique ${ }^{7}$, et très vite diffusé dans les cercles socialistes du Nord et de la Belgique ${ }^{8}$, avant d'être exécuté par les fanfares socialistes de Lille au $14^{\mathrm{e}}$ congrès du POF le 23 juillet $1896^{9}$.

On voit que, dès cette reprise populaire du chant complet, c'est bien la musique qui a lancé l'Internationale. On ne signale en effet à son propos aucune interprétation, chantée avec l'air de la Marseillaise ou un autre, avant cette date de 1888. C'est comme une greffe qui lui a fait prendre vie. Facile à mémoriser par la répétition de ses refrains, ses finales binaires, son rythme martelé, son air de type romantique, le chant allie à la perfection un texte de rassemblement communautaire et universel (« le grand parti des travailleurs ») et l'allure dynamique du langage de la marche à la fin du $19^{\mathrm{e}}$ siècle.

En quoi ces hymnes, si opposés que soient leur origine, leur milieu d'élaboration et leur rythme, le Te Deum et l'Internationale, peuvent-ils être apparentés? Bien qu'aux antipodes l'un de l'autre, ils mettent en place un nous communautaire progressivement étendu dans un espace

6. Ibid., p. 20.

7. L'Internationale, musique de Degeyter, paroles d'Eugène Pottier, Lille, imprimerie de P. Lagrange, 1889.

8. J. Milot, 1893, Recueil des chants démocratiques, Bruxelles.

9. M. Choury, 1970, p. 200. 
organisationnel à fonction idéologique et politique. Les données de la création et de la réception de ces hymnes que nous venons d'évoquer « ne sont pertinentes que sous la forme d'images, de représentations que les sujets énonciateurs s'en construisent $\gg{ }^{10}$. C'est ainsi que les marques de $1^{\mathrm{e}}$ personne plurielle doivent être envisagées comme déictiques : unités linguistiques « dont le fonctionnement sémantico-référentiel (sélection à l'encodage, interprétation au décodage) implique une prise en considération de certains des éléments constitutifs de la situation de communication, à savoir :

- le rôle que tiennent dans le procès d'énonciation les actants de l'énoncé,

- la situation spatio-temporelle du locuteur et essentiellement de l'allocutaire $»{ }^{11}$. À l'émetteur humain directement impliqué dans la $1^{\mathrm{e}}$ personne du pluriel (laudamus, confitemur, quaesumus) viennent s'ajouter une multitude d'allocutaires énonciatifs (à la $3^{\mathrm{e}}$ personne) qui convoquent devant Dieu un véritable rassemblement mystique. C'est lui qui confère au nous une dimension élargie à l'immensité des univers (ampleur omniprésente dans la répétition des totalisateurs : omnis terra, omnes Angelli, universae Potestates, Cherubim et Seraphim incessabili voce, pleni sunt caeli et terra, Apostolorum chorus, Prophetarum numerus, Martyrum exercitus, sancta Ecclesia, per orbem terrarum...). Ces deux mondes expressifs (celui des $1^{\mathrm{e}}$ et $3^{\mathrm{e}}$ personnes) convergent ensemble vers la louange en instaurant une relation communicationnelle de co-existence et d'action. Cette convergence se réalise par l'invocation vingt-trois fois répétée de la seconde personne sacrée, celle de Dieu le destinataire unique et trinitaire des deux autres ( $T u$, Te, Tibi, tuae, tuum, tuis, es, sedes, subveni, redemisti, fac numerari). L'homme s'y trouve intégré (en fait, englobant - car la $1^{\mathrm{e}}$ personne ne s'énonce explicitement que dans les deux premiers et les deux derniers versets du Te Deum), à travers l'Église, premier barreau de cette échelle de Jacob hiérarchiquement dressée vers les cieux, à l'infini de la création.

Derrière cette image mystique, se construit une parole de pouvoir. Le nous du Te Deum est idéologiquement encadré et mesuré :

- par un destinataire archi-invoqué et évoqué : le $T u$ singulier concentre sur l'unipersonnel le maximum de représentations; c'est le $T u$ majuscule de Dieu;

10. C. Kerbrat-Orecchioni, 1997, L'énonciation. De la subjectivité dans le langage, Paris, A. Colin, p. 20.

11. Ibid., p. 36 . 
- par un système de collocations (ainsi des reprises verbales : laudamus/laudat; confitemur/confitetur) qui instaure un parallélisme énonciatif dans l'action de deux partenaires en commune relation avec le même interlocuteur;

- par la continuité d'un même espace (per orbem terrarum) et d'un même temps: le présent de permanence et d'éternité rythme tous les actes de louange : pleni sunt caeli et terra majestatis gloriae tuae. Une intemporalité du nous rejoint ainsi celle de Dieu : tout est fixé. Depuis l'acte de rachat effectué au passé par le Christ (redemisti), le temps n'avance plus ${ }^{12}$. Ce nous universalisé mais encadré serait-il déjà celui des « élus »?

On dirait que le nous communautaire du Te Deum est inscrit dans un nous énonciatif de type exclusif, qui ferait partie d'une équation nous $=j e$ $+i l s-T u$, où les $i l s$ sont les garants d'une éternisation et d'une hiérarchie dans cette géométrie de la verticalité imposée à tous. Eux ne bougeant plus et Tu étant immuable (aeternum Patrem, sempiternus Filius), le nous n'a d'autre jeu possible - au sein de cette représentation immobilisante que d'obéir en confessant sa foi (confitemur) à l'exemple des autres univers, sous peine d'être exclus de l'équation. Les saints comme les prophètes sont « comptés » (c'est-à-dire inclus : numerus, numerari ).

L'analyse du nous de l'Internationale présente, elle aussi, une parole de pouvoir, mais partagé, diffusé. Ne le voit-on pas, lui aussi, cadré et mesuré?

- par la répétition de ses appellatifs et invocatifs de type emphatique ( «Sauvons-nous nous-mêmes; soufflons nous-mêmes » : hors de ce nous, pas de salut) tellement présents qu'ils se mémorisent aussi bien dans l'action que dans l'air. Ce nous multiple mais défini prend place dans toutes les strophes (à l'exception des troisième et quatrième) et se multiplie par le refrain (« Groupons-nous! »);

- par les destinataires très invoqués, très convoqués au rassemblement, à travers des formes perlocutives (4 énoncés) et exclamatives (15 signes d'exclamation), le tout entrainé dans un rythme d'accents très scandés ;

12. «Schématiquement, le temps avance avec le passé simple et n'avance plus avec l'imparfait... Schématiquement, le temps avance avec les accomplissements et les achèvements (à savoir avec les évènements) et n'avance pas avec les états et les activités (à savoir avec les états) » (A. Reboul, J. Moeschler, 1998, Pragmatique du discours. De l'interprétation de l'énoncé à l'interprétation du discours, Paris, A. Colin, p. 115). 
- par les enchainements globalisateurs et intégrateurs des destinataires sans cesse appelés à constituer ce je pluriel unifié (le " genre humain »). Celui-ci en devient très référentiel : les destinataires nommés s'y distribuent en cooccurrence avec le nous dans les mêmes couplets : « damnés..., forçats... faisons; foule esclave... soyons tout; producteurs, sauvons-nous; soufflons... notre forge; armées... rompons les rangs; ouvriers, paysans, nous sommes...»;

- par l'exclusion absolue des refusés à cette intégration qui déclinent tout au long du poème une représentation de l'ennemi: sauveurs suprêmes, Dieu, César, le tribun, le voleur, l'État, la loi, l'Impôt, le riche, les rois de la mine et du rail, la « bande », les rois et tyrans, ces cannibales, nos généraux, l'oisif, les corbeaux et vautours... Le « genre humain » est, au départ, coupé en deux;

- par le cadrage spatio-temporel qui instaure un état de continuité à travers la mise en œuvre des présents de l'indicatif (nous sommes) et surtout de l'impératif (faisons, soyons, sauvons, décrétons, soufflons, appliquons, rompons, groupons). L'intemporalité de l'action - urgence permanente de sa nécessité depuis toujours - contribue au rassemblement identitaire.

L'extension du nous énonciatif parait s'inscrire ici dans un nous inclusif $($ nous $=j e+t u(v o u s)-i l s)$ très répété. C'est une géométrie de la circularité qui s'impose à l'esprit. Partie du titre, elle boucle logiquement et périodiquement sur la phrase conclusive : «L'Internationale sera le genre humain ». Mais comme la verticalité imposait une fermeture, la circularité en impose une aussi.

Dans l'un comme dans l'autre hymne, on constate en effet que, non référentiel au départ, le nous pluriel devient un nous communautaire, identitaire et défini (par rapport à d'autres, nommés ou non), tout en s'inscrivant dans deux géométries différentes porteuses d'une représentation du monde et de l'homme. Ces deux chants sont aussi porteurs d'une parole de foi, d'un crédo par rapport à un Sauveur, béni ou refusé, et par rapport à une unification communautaire et à une action collective attendue d'elle, qu'elle soit de soumission (veneratur) ou d'opposition (Debout!). Ils portent, dans leur mystique même, une fonction politique. 


\section{Quand le nous communautaire dit le nous politique}

Une représentation mentale labellisée est à la base de cette fonction. "Idéalement, elle doit être identifiante, c'est-à-dire permettre d'isoler l'objet désigné. Elle doit par ailleurs rassembler toutes les informations sur cet objet : une étiquette qui est, en quelque sorte, le nom de la représentation mentale et qui lui est spécifique ${ }^{13}$. Du peuple des laudateurs à la foule esclave, quelle différence? L'hypothèse que nous formulons est double :

- Te Deum et Internationale mettent en œuvre des systèmes de représentation (informations conceptuelles, images, rôles, désignants) à la fois semblables et différents, qui font de ces chants des hymnes politiques;

- les référents mis en œuvre possèdent un système de valeurs et de significations qui font de ces chants des hymnes historiques, en entendant par référent « le processus de mise en relation des mécanismes qui font correspondre à certaines unités linguistiques certains éléments de la réalité extra-linguistique $\gg{ }^{14}$.

Dans le Te Deum, à partir du texte traditionnellement repris dans les missels de la liturgie, les informations conceptuelles et les expressions référentielles s'organisent en 32 versets, sans composition strophique très définie. 17 d'entre eux sont intégrés dans le chant par le pronom Tu appellatif, son accusatif $T e$ et son datif Tibi; 6 autres par l'invocation des qualifiants de cette seconde personne: Sanctus (3 fois), Dominus, Deus Sabaoth, Patrem immensae majestatis, verum et unicum Filium, Paraclitum Spiritum, Judex... Les 9 versets restants introduisent les désignants des locuteurs et énonciateurs : omnis terra, Cherubim et Seraphim; caeli et terra, sancta Ecclesia, exercitus Martyrum, credentibus... L'homme est-il inclus dans l'échelle des saints, des martyrs, des prophètes, des apôtres et des anges comme le Christ est inclus dans la gloire du Père ( $T u$ ad dexteram Dei sedes in gloria Patris)? Sans doute, mais entre l'une et l'autre réalité mystique, il n'y a pas de commune mesure : à la multiplicité des cieux et des terres qui foisonnent répond l'unicité du $T u$ divin et de la prière, plus supplicative qu'impérative : « subveni », «fac numerari ». Le Pouvoir trinitaire est en haut, dominant et séparé, hors échelle, absolu. Le rôle dévolu à la création, des hommes aux anges, est alors de reconnaitre ce $T u$, faiseur et garant d'existence, d'ordre, de justice et de salut pour la

13. Ibid., p. 134.

14. C. Kerbrat-Orecchioni, 1997, p. 34. 
communauté qui le loue. Et c'est par rapport à lui seul que le nous communautaire prend sens et identité, par rapport donc à une représentation du Pouvoir souverain comme «majesté immense », unifiée, apothéosée, intemporelle, universelle, et finaliste pour ce qui est de l'organisation de la cité des hommes et de celle des cieux. Le Te Deum, chant de pouvoir, préside ainsi à la cérémonie de la parole communautaire de ceux qui croient (credentibus) et espèrent le salut (redimisti) dans une représentation totalisatrice du divin. Le présent des verbes instaure une éternisation. L'homme y figure transfiguré, mais au bas du Pouvoir.

Dans l'Internationale, les informations conceptuelles et les expressions référentielles s'ordonnent dans une composition de 6 strophes de 8 vers suivies d'un refrain de 4 vers; d'où la répétitivité circulaire d'une strophe à l'autre, avec le retour des mêmes rimes (-ale et -ain). Les référents qui se rapportent au nous communautaire s'égrènent, pour l'essentiel, tout au long des strophes, spécifiant, couplet après couplet, une définition sociale et expansive de ce nous :

- dans le cadre d'une antinomie et d'un renversement: "Nous ne sommes rien. Soyons tout $\gg$;

- à travers des désignants très « classés » : ouvrier, paysans, le Grand Parti des Travailleurs...;

- selon le processus, là aussi, d'une transfiguration, de la foule esclave à l'Internationale du genre humain. Le nous communautaire devient un nous de rassemblement victorieux, mystiquement unifiant. Les impératifs et les futurs des verbes (de faisons à soyons, de va changer à sera) instaurent, là aussi, une sorte d'éternisation ( « Le soleil brillera toujours »), mais dans l'avenir («l'éruption de la fin »). Comme le Te Deum, l'Internationale est un chant du Salut de l'homme («Décrétons le salut commun!»).

À la mobilisation des croyants fait écho celle des militants. C'est l'espoir qui fait la clôture du chant (speravimus, speravi répète le Miserere ajouté au Te Deum; " Demain, l'Internationale sera le genre humain » affirment les répétitions du refrain dans le chant révolutionnaire).

Mais la scénographie est aux antipodes. Dans l'Internationale, c'est une scénographie terrestre horizontale (terre, table, monde, base) qui se substitue à la scénographie ascensionnelle du Te Deum. S'il y a des convocations emphatiques à une verticalité (Debout! Debout!), elles sont à taille humaine (c'est la raison qui tonne), multipliant les points d'exclamation du réveil ( 9 en deux couplets) puis - seconde scène mêlée à la première - à travers la métaphore de l'éruption (et non irruption, comme dans la coquille de la première publication) du cratère que reflètent, dans 
le couplet suivant, la forge de l'homme et le cachot où la justice était enfermée. Sur une troisième scène sont convoquées des entités abstraites affrontées (Égalité, droits et devoirs contre État, Impôt, tutelle, lois). La scène suivante multiplie les tableaux concrets dénonciateurs : mine, rail, coffres-forts, fumées, armées, crosse en l'air, cannibales, balle...). Le couplet final revient à la terre des hommes; mais une transfiguration a eu lieu : les damnés, forçats et esclaves sont devenus ouvriers, paysans et travailleurs ( $\mathrm{La}$ terre n'appartient qu'aux hommes»), débarrassés des oisifs (lire capitalistes), des corbeaux (lire curés) et des vautours (lire propriétaires). Le nous rassembleur a, au fil de ces scènes, opéré le tri entre l'humain et l'inhumain, incessamment remotivé dans la lutte par les relances du refrain. Des slogans - prenant la place des prières - rythment les phases de cette lutte dénonçant le mal : "L'impôt saigne le malheureux; nul devoir ne s'impose au riche; le droit du pauvre est un mot creux... », ou célébrant le bien : "L'Égalité veut d'autres lois; Pas de devoirs sans droits... »; d'où l'importance des connecteurs pour que, si, mais si... Toujours le même balancement dévalorisations / valorisations, image métaphorisée de la lutte des classes. Il s'agit bien d'une prise de pouvoir, qui va de la négation religieuse (" pas de Sauveur suprême ») et du refus des gouvernants autoritaires ou démagogiques (« ni César ni tribun ») à l'affirmation - à leur place - du nous communautaire transformé en nous politique («Sauvons-nous nous-mêmes, Décrétons, Soufflons nous-mêmes, Battons le fer...»). L'homme doit, peut, va conquérir, reconquérir (car il en a été volé, " dévalisé ») sa propre terre, au centre du Pouvoir. À l'institution hiérarchiquement organisée et immobilisée en regard d'une Toute-puissance que présente le Te Deum, l'Internationale substitue une restitution égalitariste suscitant sa propre transcendance (dans la trinité-identité du nous, de l'Internationale et du genre humain). Deux imaginaires aussi rassembleurs (communautaristes et unificateurs), poétiques (métaphoriques et rythmiques), utopistes (éternisateurs et apothéosiques), enfin idéologiques (motivants et moralisateurs) l'un que l'autre.

Ainsi, le nous communautaire du Te Deum et celui de l'Internationale déploient un discours de foi et de pouvoir

- qui active et mobilise dans son énonciation des mécanismes identitaires socialisés de valeur universelle, voir intemporelle et finaliste, qui ont fait que ces chants ont sauté les frontières géographiques et historiques;

- qui organise par son énonciation deux systèmes de référents et de représentation du pouvoir politique, qui garantissent la souveraineté de 
l'existence, de la justice, de l'ordre et du salut de la communauté, tout en les inscrivant dans deux géométries différentes qui les justifient au sein d'une logique de pensée.

La musique, à l'image du message, rend sensible le mouvement d'adhésion requis, entre la paix des obéissants et la lutte des révoltés... Qu'en dit l'Histoire?

\section{Quand la parole d'un pouvoir s'empare du nous politique de la communauté}

À travers quels mécanismes Te Deum et Internationale se sont-ils imposés comme deux grands hymnes politiques? La première réponse est donnée par un constat historique, celui de l'association identitaire, la quasi-assimilation, entre ces deux chants et deux grandes représentations extrêmes du pouvoir politique : les Monarchies dites « de droit divin » et les Démocraties dites «populaires». Le fondement et l'explication de cette association se trouvent dans le fait que ces chants, comme nous venons de le voir, mettent en œuvre les représentations qui justifient et sécurisent idéologiquement la politique, en entendant par là « la gestion de la vie collective, la régulation de la cité et sa défense, en un mot l'art de gouverner des citoyens regroupés. Ce sens originel, à travers les mots réunit, ensemble, collective et regroupés, puis gestion, régulation et gouverner, met l'accent sur la politique en tant que pouvoir dans l'organisation du social $\gg{ }^{15}$.

C'est ainsi que le Te Deum a été mis au service d'une politique. Sa démarche d'utilisation suit d'abord l'élaboration de la liturgie romaine : l'hymne accompagne la cérémonie de consécration des papes, des évêques et des abbés, puis des rois, des reines, des empereurs. Cet usage s'institutionnalise ecclésiastiquement dans le Liber Pontificalis ou Pontificale Romanum, qui prescrit et décrit les façons et formules liturgiques dans la célébration de la messe et le don des sacrements. Au moment de l'apparition de l'imprimerie, l'institutionnalisation s'affermit et s'étend avec la première édition de ce Liber Pontificalis (1485); puis de nouvelles éditions ${ }^{16}$ unifient et homogénéisent les rituels. Dans toutes ces éditions,

15. S. Bonnafous, M. Tournier, 1995, « Analyse du discours, lexicométrie, communication et politique », Langages, 117, p. 67.

16. 1596 sous le pape Clément VIII; 1664 sous Urbain VIII ; 1752 sous Benedicte XIV, en attendant la rénovation de Léon XIII. Nous avons utilisé pour cette analyse l'édition de 
le Te Deum est présenté comme participatif au sacre royal : il est ainsi, très directement et officiellement, mis en place dans le champ politique. C'est donc à la fin du Moyen-Âge et au début des Temps modernes, quand la Monarchie n'a plus rien eu à voir avec l'élection populaire mais est devenue une institution héréditaire et/ou uniquement sacrale, que le cérémonial a pris son importance et que le Te Deum est devenu l'hymne du prestige et de l'honneur face à la communauté à gouverner. Les cérémonies du couronnement se configurent alors en suivant les prescriptions du Liber Pontificalis, dont les Ordines de différentes cathédrales accommodent et précisent les modalités ${ }^{17}$. Et cela va jusqu'à l'intégration complète de l'hymne dans le rituel du couronnement, ainsi que le montre l'extrait suivant traduit du Pontificale Romanum : "Ensuite, le Métropolite, après avoir déposé sa mitre, debout devant l'autel entonne, suivi par le chœur, l'Hymne Te Deum laudamus. Tous reprennent le chant. Puis le Métropolite se range à la droite du Roi et y reste jusqu'à la fin de l'hymne ${ }^{18}{ }^{18}$. C'est ainsi que le chant sacré initié par le prêtre, repris par le chœur, puis élargi au roi et aux dignitaires ecclésiastiques et royaux, homogénéise et unifie la communauté chantante dans la verticalité de sa hiérarchie. La parole du Te Deum non seulement s'articule avec les gestes et les symboles, mais elle les enveloppe et les rend signifiants. Signe en arc-en-ciel de la ligne du passage du ciel à la terre, la mitre posée sur la tête du métropolite le présente comme possesseur du pouvoir; déposée, elle symbolise la «delegatio » faite au roi de la souveraineté sur la cité des hommes. Le chant est là pour rappeler en permanence d'où vient cette delegatio. Lorsque le métropolite prend place à la droite du roi, côté bénéfique et honorifique dans la tradition occidentale, il lui demande l'exercice de la justice, fonction essentielle et premier fondement de la monarchie et le $T e$ Deum, en contrepoint, rappelle l'exigence de Dieu premier servi, seul délégateur de pouvoir (per auctoritatem omnipotentis Dei, a dit le prélat au cours de l'intronisation). Le roi devient alors, à son tour, médiateur entre Dieu et les hommes; et les symboles convergent vers cette fonction : le trône, centre établi entre la coupole courbe, qui le surmonte et représente le ciel, et le thalamum, estrade carrée symbolisant la terre; le sceptre attribut du pouvoir donné sur les sujets du royaume de la part de Dieu,

1752 (voir Enciclopedia de la religion Catolica, Barcelona, 1954), qui se trouve dans la cathédrale d'Oviedo, Espagne.

17. Voir 1985, Le sacre des rois. Actes du Colloque international d'histoire sur les sacres et couronnements royaux (Reims 1975), Paris.

18. Pontificale Romanum, éd. de 1758, p. 171. 
qu'il désigne pointé vers le haut; enfin la couronne qui est lumière divine et puissance royale ${ }^{19}$. Cette cérémonie de la délégation de pouvoir fonde l'institution monarchique. La reprise en plain-chant, d'une seule voix, par toute la communauté rassemblée sonne comme une reconnaissance de l'« élection » divine. Il y a donc association identitaire entre Te Deum, communauté et roi, dans ce moment du couronnement.

L'analyse historique explique les transformations d'un rituel aussi fixe. Ainsi, la mise en musique polyphonique et orchestrale a eu pour but et conséquence d'amplifier la sonorisation de l'hymne. Réduite au début, la musique a pris peu à peu le pas sur un texte latin de moins en moins connu; c'est elle qui devient porte-parole de Dieu, qu'il s'agisse des $T e$ Deum de Taverner au $16^{\mathrm{e}}$ siècle, de Charpentier ou de Colonna au $17^{\mathrm{e}}$, de Bernier, Bernabei ou Blanchard au $18^{\mathrm{e}}$, puis de Haendel, Gossec, Berlioz, et de bien d'autres ${ }^{20}$. Leurs innovations se greffent aussi sur différents systèmes de référence, tel le Te Deum de Taverner ${ }^{21}$ composé pour les messes-parodies qui se sont célébrées dans l'Angleterre d'Henri VIII après sa rupture avec l'Église de Rome en 1534, ou encore le Te Deum de Gossec composé pour la première fête révolutionnaire du 14 juillet 1790 au Champ-de-Mars. Dans ces deux cas, le Te Deum, non parodique en luimême, rend compte de la solennité du pouvoir (Henri VIII se passe de pape mais ni de Dieu ni d'Église) et de la force du nous communautaire politique (la «Fédération » révolutionnaire célèbre son unité sur l'" autel » de la Patrie). Évidemment non parodique aussi, ce Te Deum qui a retenti dans toutes les Églises de France à la Libération en 1945 : il s'agissait de remerciement, mais aussi de la reconnaissance d'un Pouvoir relégitimé et sécurisant, garant d'existence et de salut pour la communauté à nouveau regroupée dans le système référentiel de l'Église.

Le rejet, l'exécration de l'usage politique du Te Deum n'est pas moins symbolique. On en trouve un excellent exemple dans le poème des Châtiments intitulé justement «Te Deum ». Victor Hugo accuse Monseigneur Sibour, archevêque de Paris, de s'être vendu à Napoléon le Petit (pour un siège de sénateur), en célébrant un Te Deum solennel, à sa demande, dans Notre-Dame le $1^{\text {er }}$ janvier 1852. Il s'agissait pour Louis-Napoléon de se poser en égal de son oncle, qui fut couronné par le pape. Ce Te Deum le lavait du coup d'État et le sacralisait. Pour Hugo, ses référents étaient les morts du 2 décembre précédent :

19. L. Benoist, 1975, Signes, symboles et mythes, Paris, PUF, p. 48, 55.

20. P. Huot-Pleuroux, 1957, Histoire de la musique religieuse, Paris, PUF.

21. Ibid., p. 93. 
Prêtre, ta messe, écho des feux de peloton

Est une chose impie.

Derrière toi, le bras ployé sous le menton,

Rit la mort acccroupie.

Ce Te Deum sacrilège devient un hymne à l'envers, référents retournés :

Prêtre, on voit frissonner, aux cieux d'où nous venons,

Les anges et les vierges,

Quand un évêque prend la mèche des canons

Pour allumer les cierges!

On voit ici, mise à nu par Hugo, l'utilisation idéologique du Te Deum, au service - comme l'Église officielle - des « hommes d'ordre ». Il en est de même dans l'opéra de Puccini, La Tosca (Milan, 1896) : à la dernière scène du premier acte est organisée une procession pour l'entrée du cardinal, au cours de laquelle, rassemblée autour d'un Te Deum, la foule mise en scène proclame la victoire remportée par l'armée du roi de Naples et de l' "Autrichienne » sur les "patriotes », républicains partisans de Napoléon. L'acclamation rituelle «Te Deum laudamus » et les cris de « Viva il Re!» se mêlent intimement.

De son côté l'étude des usages de l'Internationale met à jour son appropriation et son utilisation par les organisations socialistes et communistes. Cela n'est pas allé sans querelles et drames. D'inspiration plutôt anarchiste anti-autoritaire chez Eugène Pottier, adepte du Grand Soir, antimilitariste (Ni Dieu, ni César, ni tribun; le monde va changer de base; c'est l'éruption de la fin; L'État comprime et la loi triche; crosse en l'air et rompons les rangs; nos balles sont pour nos propres généraux) ${ }^{22}$, la chanson relancée par la musique de Pierre Degeyter s'étend aux cercles syndicaux et socialistes du Nord puis de Belgique et de France dès la fin du siècle. Elle appartient à ceux qui la chantent. Ce qui entraine luttes internes et polémiques, qui vont jusqu'au tribunal à propos d'une dispute

22. Une version carrément anarchiste invoque les «Fiers compagnons » et prône l'Égalité des droits seule (L'Égalité n'a pas de lois/Égaux, nous n'avons que des droits!). Voir P. Brochon, 1966, p. 101, notes. De même, au début du $19^{\text {e }}$ siècle, se chante en Espagne une Internationale anarchiste. D'autres Internationales " particularisent » certaines communautés en introduisant quelques changements dans le texte, telles celle des viticulteurs du Languedoc en 1907 ou celle des éleveurs en 1911, qu'on a appelée « L'Internationale du beurre ». 
sur les droits d'auteur de la musique, cédés par Adolphe Degeyter, le chanteur, au Parti socialiste français. Une lettre d'Amédée Dunois à Roger Salengro, maire de Lille, se fait, à plusieurs années de là, l'écho de cette dispute entre frères, qui recouvre une lutte entre possibilistes et collectivistes, socialistes modérés et révolutionnaires : "Nous sommes, écrit Dunois, en présence de deux thèses, l'une socialiste favorable à Adolphe, 1'autre communiste qui tient pour Pierre $\gg{ }^{23}$.

Hymne de la Seconde Internationale réunie à Bruxelles en aout 1891 puis surtout au Congrès de Copenhague de 1910 qui l'officialise comme « internationale », l'hymne révolutionnaire se voit traduit et chanté dans toutes les langues. «Cette immortelle Internationale, qui [constate dès 1910 E. Museux ${ }^{24}$ ] est devenue la Marseillaise des Travailleurs du monde entier », est publiée en roumain en 1900, en russe en 1902 (et chantée pendant la révolte de 1905), en finnois en 1906, en allemand en 1924, en japonais et en bulgare en 1949, etc. Après février 1917, plus de 700 éditions ont paru en russe, parfois même sans le nom des auteurs, en tant qu' «hymne du Parti » ${ }^{25}$. On peut s'interroger sur ce que deviennent les paroles libertaires d'Eugène Pottier dans les innombrables adaptations «nationales » qui en sont faites. L'Internationale n'est-elle pas sacrée hymne officiel de l'URSS en 1918, à l'instigation du Soviet de Pétrograd?

Par la suite, la confusion entretenue par l'Osvag ${ }^{26}$ et le Komintern entre cet usage proprement soviétique et son usage international poussera dans le sens d'une identification de l'URSS et de sa défense à la cause communiste tout entière. Confusion devenant dogme ou bien violemment refusée : le socialisme international, déjà déchiré en 1889 (avec les deux congrès rivaux de Paris), se redéchirant après la révolution d'octobre 1917, c'est bien entre deux partis politiques (coupés en France entre SFIO et SFIC au congrès de Tours de 1920) qu'une rivalité s'instaure dans l'usage de L'Internationale. En 1924, à un mois d'intervalle, c'est avec ce même hymne que le congrès du PS-SFIO vote une protestation contre la

23. M. Ferro, 1996, p. 32. Texte du 18 décembre 1927 dans J. Tint, 1976, « L'Internationale, musique de Pierre Degeyter », La Commune, Revue d'Histoire de l'Association des Amis de la Commune de Paris, 4, p. 43-66. Fin de la querelle fratricide : Adolphe Degeyter s'est suicidé en février 1916.

24. E. Museux, p. 43.

25. P. Brochon, p. 228-241.

26. Semi-sigle composé d' "Information » et "Agitation », nom du ministère de la propagande soviétique (voir S. Tchakhotine, 1952, Le viol des foules par la propagande politique, Paris, Gallimard NRF, p. 329-338). 
«persécution des sociaux-démocrates et des socialistes révolutionnaires russes qui combattent le régime bolcheviste » et que le congrès du PCSFIC vote un appel aux travailleurs où il proclame : "Seule la République soviétique, livrée au travail pacifique et constructeur, donne au monde l'exemple de la prospérité, de la création, de la vraie grandeur ». Les deux «Internationales » politiques (la Seconde, socialiste, et la Troisième, communiste) s'en revendiqueront également. Leurs congrès l'incluent dans des rituels qui deviennent immuables: solennité (délégués tous debout, démarrage dans un silence), poing droit dressé, signe de force et d'union. D'autres signes différencient les identités : le drapeau rouge marqué de la faucille et du marteau (symbole bolchévique) a fait partie du cérémonial communiste, alors que les socialistes et socio-démocrates le refusaient, préférant agiter dans leurs manifestations leur drapeau rouge aux trois flèches ${ }^{27}$.

Mais l'hymne fait l'objet d'une appropriation grandissante du côté du PCF ; ne servirait-il pas à montrer une affirmation de présence, de force, de prépondérance, en même temps qu'il répondrait à une tactique d'englobement? Avec lui, le PCF «marque » les manifestations populaires, y compris celles des autres; il leur impose son sceau. En novembre 1924, au transfert des cendres de Jaurès au Panthéon, la manifestation officielle silencieuse est suivie d'une seconde, où 100000 communistes entonnent l'Internationale. Onze ans plus tard, il suffit qu'Edouard Herriot apparaisse à une tribune lyonnaise célébrant l'amitié France-URSS pour qu'il soit salué de la même manière; quelques mois plus tôt, Léon Blum et Maurice Thorez se serraient les mains aux accents de l'Internationale chantée debout au Palais des Sports ${ }^{28}$.

Cette année-là pourtant le « grand tournant » patriotique du PCF met un premier bémol à cette utilisation stratégique d'un hymne ressenti soudain comme (un peu) trop internationaliste. En juillet 1935, en effet, au stade Buffalo, Jacques Duclos sous des drapeaux tricolores mêlés aux drapeaux rouges s'écrie : "Si l'immense foule rassemblée aujourd'hui chante en cette mémorable journée, non seulement notre hymne d'espérance et de lutte, l'Internationale, mais aussi La Marseillaise, nous n'oublierons pas que La Marseillaise est un chant révolutionnaire... ${ }^{29}$.

27. Symbole majeur de la résistance du «Front d'airain » au nazisme (voir S. Tchakhotine, 1952, p. 374-418).

28. J.-J. Becker, S. Berstein, 1987, Histoire de l'anti-communisme en France, Paris, Orban, p. 186, 257, 296.

29. Ibid., p. 245. 
Fusion symbolique, à travers les chansons et les drapeaux, du nationalisme et de l'internationalisme. Aucun signe immergé dans la société et dans l'histoire ne se cantonne donc à sa valeur idéologique : disputée, annexée, concurrencée, l'Internationale n'a pas échappé à sa fonction politique. Comme le Te Deum, elle a été au service de stratégies de pouvoir.

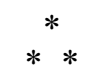

Avec la chute des rois et la désacralisation des monarchies populaires ou constitutionnelles (ou des républiques patriciennes), le Te Deum se fait rare. Il reste parfois en tant que symbole rattaché au passé d'un pays. De même, on constate de nos jours que l'Internationale ne reste opérante que dans un champ progressivement réduit; le premier indicateur de cette réduction remonte à décembre 1943, date à laquelle l'hymne soviétique disparait au profit d'un hymne officiel russe, le chant de L'Union indestructible des libres Républiques. Le Sovnarkon, conseil des commissaires $\mathrm{du}$ peuple, explique cette substitution en affirmant que l'Internationale « ne répondait plus aux changements profonds qui s'étaient produits dans le pays et n'exprimait plus la réalité vraie de ce qui était le socialisme en URSS $»^{30}$.

Cette réduction progressive du Te Deum comme de l'Internationale à l'état de symboles historiques est-elle le signe, en pleine expansion d'une " globalisation » économique sans contrôle, de la disconvenance de ces chants universalistes par rapport soit à la non-transparence voire à la dissimulation des pouvoirs d'argent et des ambitions de profit, soit au repli sur des continents, des pays, des régions ou des ethnies? Ces hymnes, francs comme l'or dans leur idéologie, ne sont au niveau ni de la mondialisation ni des particularismes d'aujourd'hui. Cela signifie-t-il qu'il n'y a plus de place, au terme d'histoires désidéologisées, pour la parole communautaire de l'homme universel?

30. M. Ferro, 1996, p. 71. 